\title{
Phosphorite deposition and Earth system oxygenation in the Paleoproterozoic Era
}

\author{
Michael KIPP ${ }^{1}$, Aivo LePLAND ${ }^{2}$, ROGER BUICK ${ }^{3}$ \\ ${ }^{1}$ Division of Geological \& Planetary Sciences, California \\ Institute of Technology (mkipp@ caltech.edu) \\ ${ }^{2}$ Geological Survey of Norway \\ ${ }^{3}$ Department of Earth \& Space Sciences, University of \\ Washington
}

Although there is broad consensus about the first-order trajectory of ocean oxygenation across the Great Oxidation Event (GOE), the cycling of major nutrients (i.e. nitrogen \& phosphorus) at this time remains a topic of debate. Phosphorus (P) is particularly important in this context, since it is thought to set the productivity of the marine biosphere on geological timescales, with $\mathrm{P}$ scarcity possibly leading to a smaller, prokaryotedominated biosphere. While it is known that $\mathrm{P}$ cycling is closely tied to redox chemistry in the modern ocean, this has yet to manifest in a clear understanding of how ocean oxygenation across the GOE affected the $\mathrm{P}$ cycle. Here we review mechanisms that have been proposed for Archean P limitation and contrast their implications for P cycling across the GOE. Then we turn to the geologic record and assess $\mathrm{P}$ cycling in a marine setting that contains some of the oldest P-enriched marine sediments in the geologic record: the $2.0 \mathrm{Ga}$ Zaonega Formation (ZF) in Karelia, Russia. New geochemical data suggest that sedimentary $\mathrm{P}$ enrichment occurred in the ZF during intervals of basinal connection to the open ocean, resulting in a supply of oxidants, namely sulfate, that fueled remineralization reactions and created porewater conditions permissive of authigenic phosphate precipitation. Looking beyond the ZF, we ponder the broader relationship between authigenic phosphorus burial and ocean oxygenation, positing that the temporal distribution of phosphorites may track the growth of the marine oxidant reservoir across Earth's history. 